
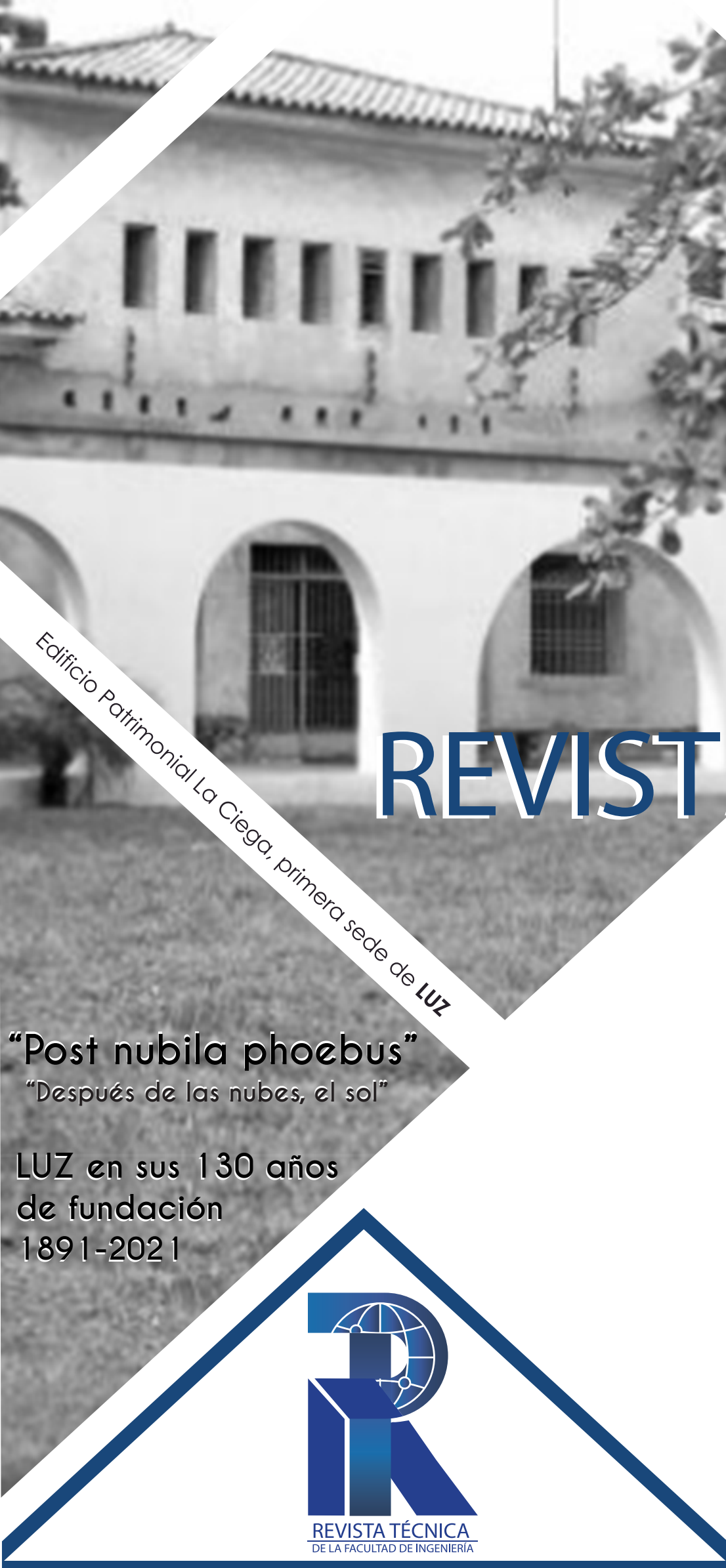

, $18,4,2,1$
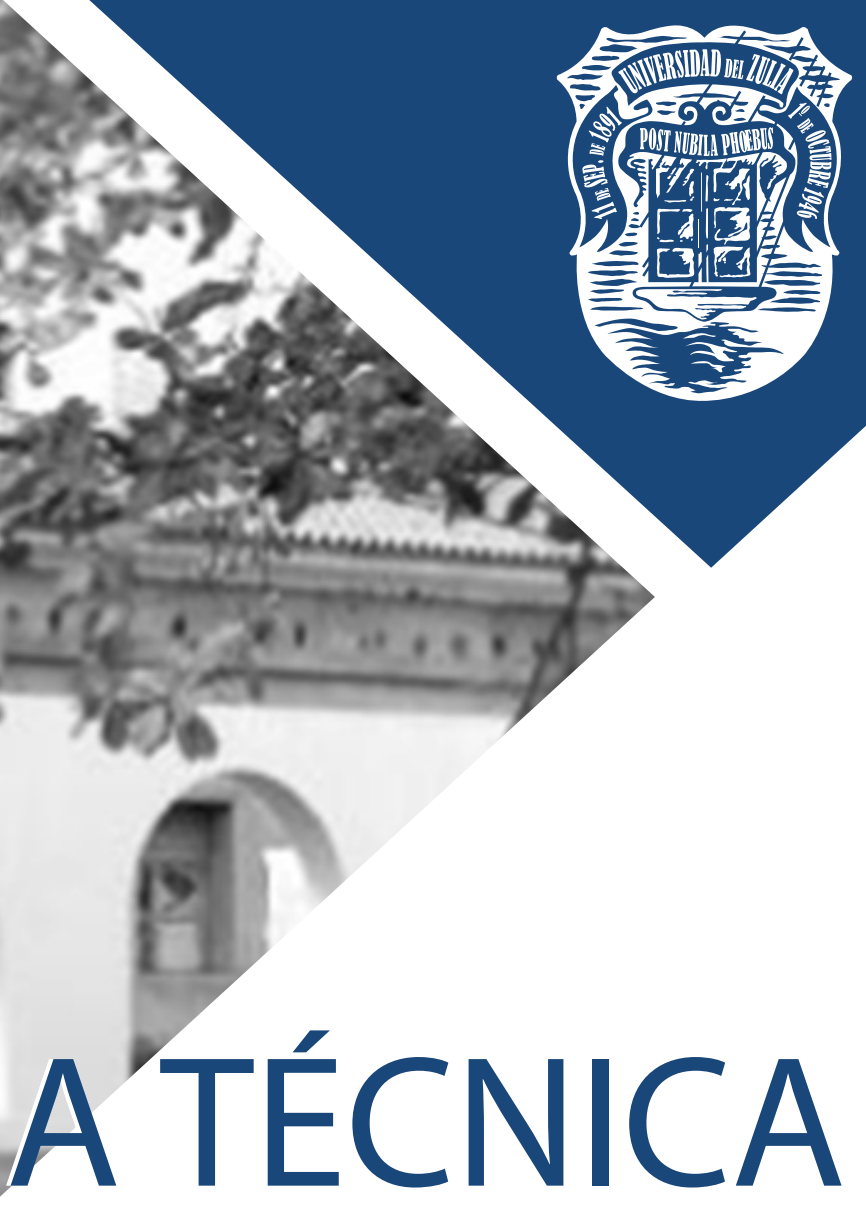
DE LA FACULTAD DE INGENIERÍA

Una Revista Internacional Arbitrada que está indizada en las publicaciones de referencia y comentarios:

- SCOPUS

- SCIELO

- LATINDEX

- DOAJ

- MIAR

- REDIB

- AEROSPACE DATABASE

- CIVIL ENGINEERING ABTRACTS

- METADEX

- COMMUNICATION ABSTRACTS

- ZENTRALBLATT MATH, ZBMATH

- ACTUALIDAD IBEROAMERICANA

- BIBLAT

- PERIODICA

- REVENCYT

UNIVERSIDAD DEL ZULIA 
Rev. Téc. Ing. Univ. Zulia. Vol. 44, No. 2, Mayo-Agosto, 2021, 104-116

\title{
Análisis técnico-económico de naves industriales mediante interpolación no lineal de Lagrange.
}

\author{
Diego Fernando Loachamin-Chano ${ }^{1}(\mathbb{D})$, Andrea Elizabeth Freire-Luna ${ }^{1}(\mathbb{D}$, \\ David Patricio Guerrero-Cuasapaz ${ }^{1 *} \mathbb{D}^{\mathbb{D}}$, Milton Bolívar Guerrón-Figueroa $^{1}$ (D)
}

${ }^{1}$ Facultad de Ingeniería Civil, Universidad Politécnica Salesiana. Quito, Ecuador.

*Autor de correspondencia: dguerrero@ups.edu.ec

https://doi.org/10.22209/rt.v44n2a05

Recepción: 08 de septiembre de 2020 | Aceptación: 12 de febrero de 2021 | Publicación: 15 de abril de 2021

\section{Resumen}

Debido al notable incremento de construcciones de naves industriales en Ecuador, es necesario realizar un análisis técnico-económico para conocer la incidencia del costo de materiales sobre ellas. Para ello, se elaboró una muestra de 40 naves, fijando relación entre luz libre (L), altura del pórtico (H) y distancia entre pórticos (B), con el propósito de determinar una métrica. Para este estudio se estableció el peso de la estructura en $\mathrm{kg} / \mathrm{m}^{2}$. Con los resultados se aplicó un modelo matemático (interpolación de Lagrange), se obtuvo un polinomio que describe el modelo de cada grupo de naves y su representación gráfica, por consiguiente, se determinó una aproximación del peso de estructuras. Las métricas que se hallaron tienen una repercusión directa en el costo de los materiales de las estructuras, estas se obtuvieron al multiplicarlas por el costo del kg de acero. Se realizó una comprobación con una muestra de 6 naves, en las se halló el peso utilizando el diseño por factores de carga y resistencia y luego con el polinomio; obteniéndose un error: para naves moduladas a $5 \mathrm{~m}$ una media de 0,03 \% con una desviación de 0,03 y para naves moduladas a $6 \mathrm{~m}$, una media de 0,07 \% con una desviación de 0,03.

Palabras clave: ábacos; interpolación; Lagrange; naves industriales; pesos.

\section{Technical-economic analysis of industrial buildings using non-linear Lagrange interpolation.}

\begin{abstract}
Due to the notable increase in industrial buildings in Ecuador, it is necessary to carry out a technical-economic analysis to know the impact of the cost of materials on them. To do this, a sample of 40 bays was drawn up, establishing the relationship between free span (L), portico height $(\mathrm{H})$ and distance between porticoes (B), in order to determine a metric. For this study, the weight of the structure was established in $\mathrm{kg} / \mathrm{m}^{2}$. With the results, a mathematical model was applied (Lagrange interpolation), a polynomial was obtained that describes the model of each group of ships and its graphic representation, therefore, an approximation of the weight of structures was determined. The metrics that were found have a direct impact on the cost of the materials of the structures, these were obtained by multiplying them by the cost of the kg of steel. A check was carried out with a sample of 6 ships, in which the weight was found using the design by load and resistance factors and then with the polynomial; obtaining an error: for ships modulated at $5 \mathrm{~m}$ an average of $0.03 \%$ with a deviation of 0.03 and for ships modulated at $6 \mathrm{~m}$, an average of $0.07 \%$ with a deviation of 0.03 .
\end{abstract}

Keywords: abacus; interpolation; Lagrange; industrial buildings; weight. 


\section{Introducción}

Las naves industriales son edificaciones que permiten no solo el almacenamiento de víveres, sino también son utilizadas para centros de elaboración, túneles de lavado, oficinas, centros comerciales, industrias, etc. (Hernández, 2015), los cuales permiten la satisfacción del hombre en varias esferas de la sociedad. Una nave industrial es un edificio de uso industrial que alberga la producción y/o almacena los bienes industriales, junto con los obreros, las máquinas que los generan, el transporte interno, la salida y entrada de mercancías, entre otros (Arnal et al., 2014).

El diseño de naves industriales en Ecuador tiene como base la Norma Ecuatoriana de la Construcción (NEC, 2014), la cual se basa en normativas tales como: ASCE (2017), AISC (2016) y AISI (2016), las cuales se dedican a estudiar las características y formas del comportamiento del acero y la elaboración de normas para cálculo estructural que rigen el diseño en acero, desarrollándose así el método de estado límite, diseño por factores de carga y resistencia (DFCR) (NEC, 2014; AISC, 2016; AISI 2016; ASCE, 2017; Cano y Imanpour, 2020).

En la ingeniería civil y más concretamente en el campo de la construcción es fundamental tener un presupuesto bien estructurado para la correcta ejecución de una estructura; es conocido que no siempre se puede disponer de cantidades de obra sin antes haber realizado un análisis y diseño sismorresistente de una edificación, tal es el caso de las naves industriales (Guerrero, 2019). En consecuencia, en la presente investigación se planteó realizar un análisis y diseño estructural de un grupo de naves industriales que tienen características particulares en cuanto a longitud, altura y espaciamiento entre pórticos. Del análisis se obtuvieron como referencia los pesos sobre $\mathrm{m}^{2}$ de cada nave, que a su vez sirvió para aplicar una interpolación no lineal de Lagrange, lo que conllevó a determinar una ecuación con su respectiva representación gráfica (ábacos); con estos resultados se logró determinar de una forma bastante aproximada el peso sobre $\mathrm{m}^{2}$ de naves industriales diferentes a las que se tomó en principio de la investigación, para posteriormente con estos resultados hallar el costo del material que interviene en cada estructura.

\section{Materiales y Métodos}

El objetivo fundamental de esta investigación fue realizar un análisis técnico de un grupo de naves industriales, para posteriormente encontrar los costos de material de acero con la utilización de interpolación no lineal de Lagrange, que se representa a través de ábacos.

\section{Prediseño de elementos estructurales de las naves industriales}

Una de las consideraciones que se tomaron en esta investigación fue un prediseño de los elementos estructurales que constituyen las naves industriales, esto con el objetivo de iniciar con datos que se aproximarán a los resultados esperados en el comportamiento de un pórtico bajo solicitaciones gravitacionales, que debe cumplir los requerimientos de resistencia. Generalmente, se colocan refuerzos en las zonas con mayores esfuerzos para asegurar la estabilidad y seguridad de la estructura (Élez, 2016). De esta manera específica, la investigación tuvo como punto de partida los parámetros: altura libre de columna (H), luz libre de la nave (L), modulación entre pórticos (B1 o B2), definidos en la Tabla 1 e ilustrados en la Figura 1; con ello se definió la geometría de la estructura, obteniendo 40 combinaciones de naves industriales, las cuales fueron analizadas y modeladas.

Tabla 1. Variantes y combinaciones de altura de columna libre, ancho de los pórticos y longitud de la nave industrial.

\begin{tabular}{|c|c|c|c|c|c|c|}
\hline \multicolumn{4}{|c|}{$\begin{array}{l}\text { Altura de columna libre } \\
\text { (Hi) (m) }\end{array}$} & \multirow{2}{*}{$\begin{array}{c}\begin{array}{c}\text { Luz libre } \\
\text { (m) }\end{array} \\
\mathrm{L}\end{array}$} & \multicolumn{2}{|c|}{ Modulación (m) } \\
\hline H1 & $\mathrm{H} 2$ & $\mathrm{H} 3$ & $\mathrm{H} 4$ & & B1 & B2 \\
\hline 6 & 7 & 8 & 9 & 15 & 5 & 6 \\
\hline 6 & 7 & 8 & 9 & 20 & 5 & 6 \\
\hline 6 & 7 & 8 & 9 & 25 & 5 & 6 \\
\hline 6 & 7 & 8 & 9 & 30 & 5 & 6 \\
\hline 6 & 7 & 8 & 9 & 35 & 5 & 6 \\
\hline
\end{tabular}




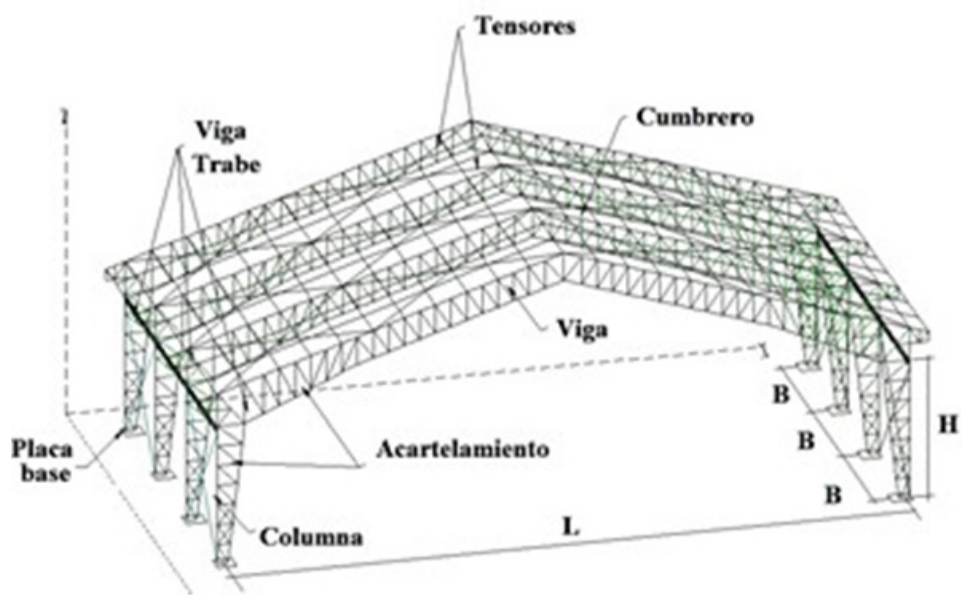

Figura 1. Geometría y variantes de nave industrial metálica.

Las naves industriales conformadas por la perfilería estructural conforme con la ASTM A 1011 (2018) son: canales tipo C, correas tipo G conformadas en frío, perfilería estructural (ASTM A36, 2018; A36M-08, 2018) como son: ángulos tipo L conformados en caliente (ASTM A1011, 2018; A1011M-18a, 2018). El presente artículo, en lo concerniente al diseño estructural, se realizó con el método de diseño por factor de carga y resistencia denominado DFCR, el cual considera la resistencia o condición de falla, donde las cargas de servicio aplicadas son factorizadas y la resistencia teórica del material es reducida. El criterio que debe satisfacer la carga factorizada deberá ser menor o igual a la resistencia reducida (Garibov y Bashirzade, 2020). Este enfoque se basa en dos conceptos fundamentales; el estado límite de resistencia y estado límite de servicio. El primero se centra en la seguridad o capacidad de carga de las estructuras e incluye las resistencias plásticas, de volteo, de fatiga, de fractura, de pandeo; mientras que los estados límites de servicio, se refieren al comportamiento de las estructuras bajo cargas normales de servicio y tienen que ver con aspectos asociados con el uso y la ocupación, tales como deflexiones excesivas, deslizamientos, vibraciones y agrietamientos (McCormac, 2013). En el método DFCR (Garibov y Bashirzade, 2020; Crisafulli, 2018), recomiendan que las cargas de servicio (Qi) se multipliquen por ciertos factores de carga o seguridad $(\lambda i)$, que son casi siempre mayores que 1,0; para obtener las "cargas factorizadas" usadas para el diseño de la estructura. Las magnitudes de los factores de carga varían, dependiendo del tipo de combinaciones de carga, como se puede apreciar en las Ecuación 1 al 6 (NEC, 2014):

$$
\begin{gathered}
U_{1}=1,4 D+L \\
U_{2}=1,2 D+1,6 L+0,5\left(L_{r} \circ S \circ R_{r}\right) \\
U_{3}=1,2 \bar{D}+1,6 L+0,5\left(L_{r} \circ S \circ R_{r}\right)+0,5(L \circ 0,8 W) \\
U_{4}=1,2 D+1,3 W+0,5 L+0,5\left(L_{r} \circ S \circ R\right) \\
U_{5}=1,2 D+1,5 E+0,2 S \\
U_{6}=0,9-(1,3 W \circ 1,5 E)
\end{gathered}
$$

Donde: $\mathrm{U}_{\mathrm{i}}$ : combinación de carga, $\mathrm{D}$ : carga muerta, L: carga viva, $\mathrm{L}_{\mathrm{r}}$ : carga viva de techo, $\mathrm{S}$ : carga de nieve, $\mathrm{R}_{\mathrm{r}}$ : carga de lluvia, excepto encharcamiento, $\mathrm{W}$ : carga de viento.

La estructura se dimensiona para que tenga una resistencia última de diseño suficiente para resistir las cargas factorizadas. Esta resistencia se considera igual a la resistencia teórica nominal (Rn) del miembro estructura, multiplicada por un factor de resistencia Ø que es normalmente menor que 1,0 (Hernández, 2015), según:

$$
\sum \lambda_{i} Q_{i} \leq \emptyset R_{n}
$$


La resistencia de diseño de cada componente estructural es mayor o igual a la resistencia requerida determinada, de acuerdo con las combinaciones de carga DFCR, de acuerdo con:

$$
R_{u} \leq \emptyset R_{n}
$$

Donde: $R_{u}$ : resistencia requerida por el método DFCR, $R_{n}$ : resistencia nominal dada por el material, $\emptyset$ : factor de resistencia, $\emptyset \mathrm{R}_{\mathrm{n}}$ : resistencia de diseño.

En esta investigación, el análisis y diseño de los elementos estructurales se realizó mediante paquetes computacionales, los cuales fueron sometidos a combinaciones de carga a cada grupo de naves industriales modulados a cada 5 y $6 \mathrm{~m}$, cada uno de ellos con altura de columna que varió entre 6 y 9 m y luz libre de 15 a 35 m, basados en la norma ecuatoriana de la construcción (NEC, 2014).

Los parámetros que intervienen en el cálculo del porcentaje de carga sísmica, que constituirán el cortante basal aplicado a cada nave industrial, se tabulan en la Tabla 2.

Tabla 2. Parámetros que intervienen en el cálculo del cortante basal de naves industriales.

\begin{tabular}{lcc}
\hline \multicolumn{1}{c}{ Parámetro } & Variable & Valor \\
\hline Zona sísmica & $\mathrm{V}$ & - \\
Factor de aceleración de la zona & $\mathrm{Z}$ & 0,4 \\
Tipo del suelo & $\mathrm{D}$ & - \\
Factor de sitio $\mathrm{F}_{\mathrm{a}}$ & $\mathrm{F}_{\mathrm{a}}$ & 1,20 \\
Factor de sitio $\mathrm{F}_{\mathrm{d}}$ & $\mathrm{F}_{\mathrm{d}}$ & 1,19 \\
Factor comportamiento inelástico del suelo & $\mathrm{F}_{\mathrm{s}}$ & 1,28 \\
Factor asociado a la ubicación geográfica del proyecto & $\mathrm{r}$ & 1 \\
Relación de amplificación espectral & $\mathrm{\eta}_{\mathrm{i}}$ & 2,48 \\
Aceleración en $\mathrm{T}=\mathrm{T}_{\mathrm{o}}$ & $\mathrm{S}_{\mathrm{a}}[\mathrm{g}]$ & 1,19 \\
Período límite en $\mathrm{T}=\mathrm{T}_{\mathrm{o}}$ & $\mathrm{T}_{\mathrm{o}}[\mathrm{s}]$ & 0,127 \\
Período límite en $\mathrm{T}=\mathrm{T}_{\mathrm{c}}$ & $\mathrm{T}_{\mathrm{c}}[\mathrm{s}]$ & 0,698 \\
Período límite en $\mathrm{T}=\mathrm{T}_{\mathrm{L}}$ & $\mathrm{T}_{\mathrm{L}}[\mathrm{s}]$ & 2,856 \\
Coeficiente $\mathrm{C}_{\mathrm{t}}$ & $\mathrm{C}_{\mathrm{t}}$ & 0,073 \\
Coeficiente para cálculo de período & $\alpha$ & 0,75 \\
Altura total del elemento & $\mathrm{h}_{\mathrm{n}}[\mathrm{m}]$ & - \\
Período teórico método 1 & $\mathrm{T}_{1}[\mathrm{~s}]$ & 0,441 \\
Período teórico método 2 & $\mathrm{T}_{2}[\mathrm{~s}]$ & 0,57 \\
Factor de reducción de fuerzas sísmicas & $\mathrm{R}_{2}$ & 3,00 \\
\hline & &
\end{tabular}

La carga viva (CV) tuvo un valor igual a la carga de granizo que, según la norma NEC SE CG (2014), por defecto, tiene un valor de peso específico del granizo igual a $1 \mathrm{~T} / \mathrm{m}^{3}$ en pendientes menores al $15 \%$ y una carga mínima de $0,5 \mathrm{kN} /$ $\mathrm{m}^{2}$. En este caso se considera la acumulación del granizo en corto tiempo y se asume un espesor de la capa de granizo de $10 \mathrm{~cm}$ de altura y un peso específico de $0,75 \mathrm{~T} / \mathrm{m} 3$; obteniendo una carga de $75 \mathrm{~kg} / \mathrm{m} 2$, la misma que se utilizó en todas las modelaciones: La carga muerta (CM) que se consideró fue de $5,0 \mathrm{~kg} / \mathrm{m} 2$ para instalaciones y de $4,0 \mathrm{~kg} / \mathrm{m} 2$ para la cubierta, teniendo un valor constante para todo el conjunto de naves industriales de $\mathrm{CM}=9 \mathrm{~kg} / \mathrm{m} 2$ y e $\mathrm{CV}=75 \mathrm{~kg} / \mathrm{m} 2, \mathrm{las}$ cuales se representan en la Figura 2 (Redroban, 2015). 


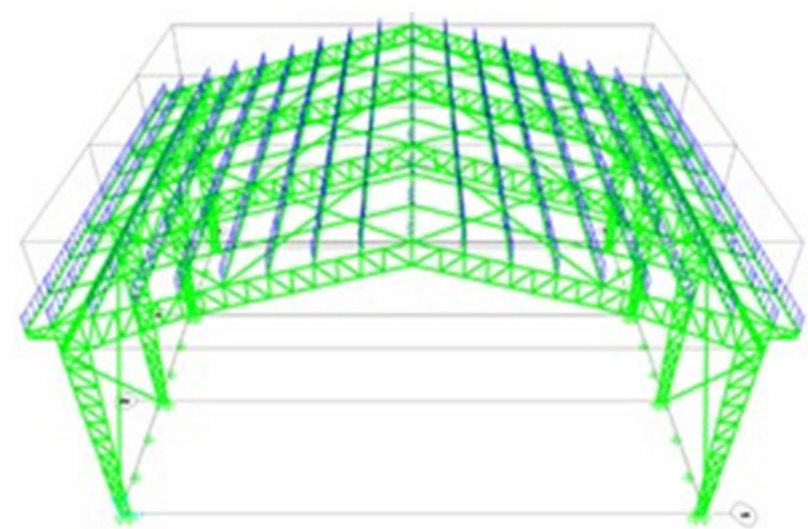

Figura 2. Carga viva y carga muerta de naves industriales.

La representación gráfica en las Figuras 3 y 4 de la carga de viento, correspondiente a barlovento W1, W2 y sotavento W3 y W4, con modulación entre pórticos a cada 5 y 6 m, respectivamente (Hernández, 2015).

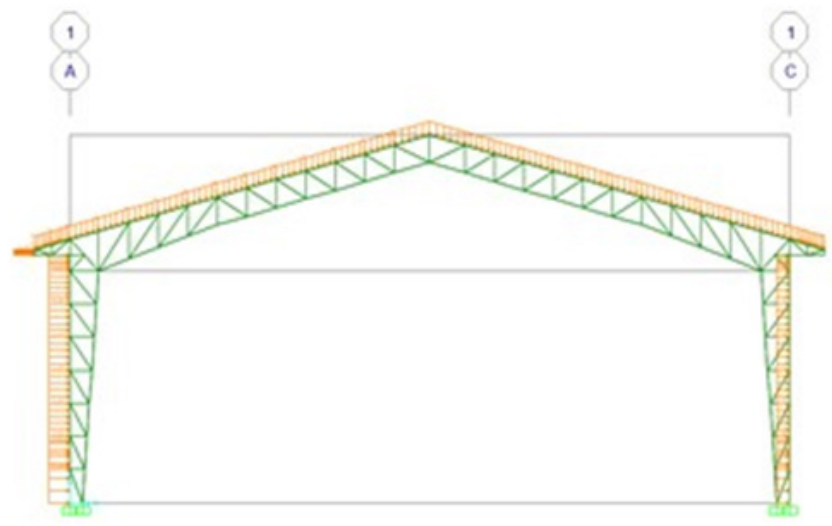

Figura 3. Carga de viento de naves industriales. W1: barlovento columna, W2: barlovento viga, W3: sotavento viga, W4: sotavento columna.

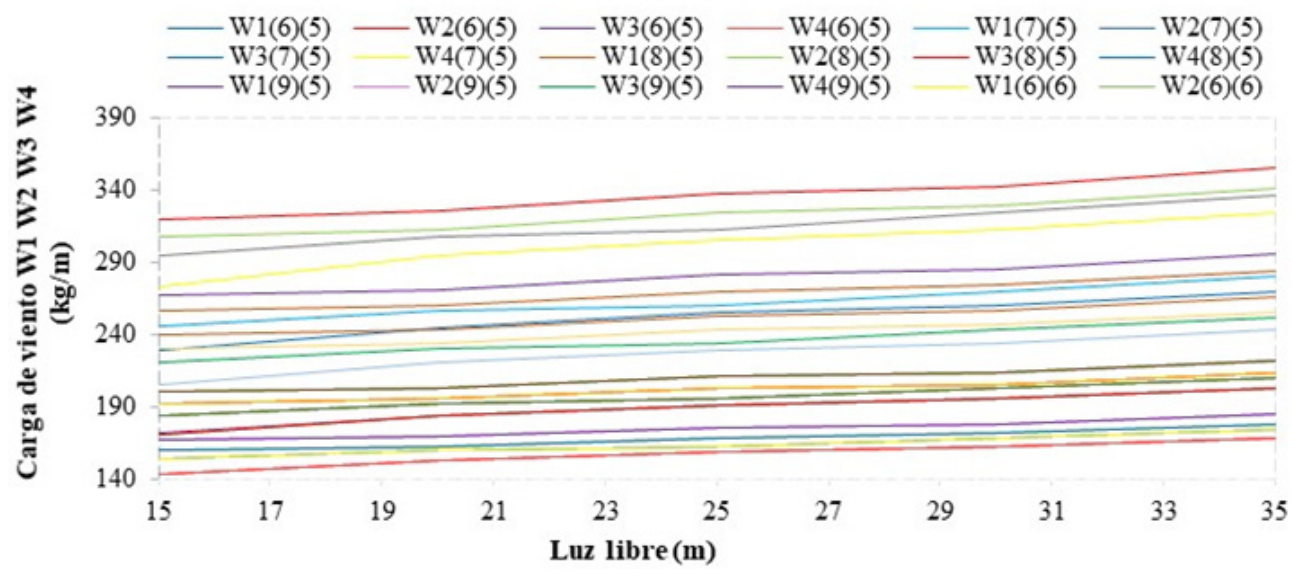

Figura 4. Resumen de cargas de viento (W1, W2, W3 y W4) para naves industriales con modulación entre pórticos B=5 y 6 $\mathrm{m}$ (barlovento). 


\section{Diseño de elementos estructurales de las naves industriales}

Para estos diseños, se verificó que cumplieran con los controles estipulados, tanto en la normativa ASCE (2017), SEI 7-16 (2017) como la NEC (2014). La optimización de las estructuras es el paso para seguir con el objetivo de garantizar las condiciones de servicio y resistencia. Para ello, se utilizó la herramienta computacional en la modelación y el análisis de las naves industriales, para determinar el peso total de todas las secciones que constituyen el galpón, de una manera sistemática (ASCE, 2017). De esta manera, se procedió a la obtención de los esfuerzos y demás solicitaciones máximas requeridas, tanto en columnas como en vigas, para con ello realizar un diseño de los elementos sujetos a compresión, verificando que se cumplieran los estados límites de pandeo local y pandeo global. De igual manera, se realizó un diseño a miembros sujetos a flexión, los cuales fueron analizados para efectos de fluencia y pandeo lateral torsional, según el manual de construcción en acero (SAP 2000, 2016; AISC, 2016).

Una vez llevado a cabo el análisis, se diseñaron los elementos estructurales, en los cuales se verificó el radio demanda-capacidad que involucran a la capacidad de compresión con la capacidad de resistir momentos flexionantes, el cual no debe ser mayor a 1,0 (Cuichan, 2016).

En esta investigación la mayoría de los elementos se encontraron entre 0 y $95 \%$ de su capacidad, es decir, que se encontraban trabajando de una manera muy aceptable, ya que en estructuras metálicas se debe procurar que trabajen a su máxima capacidad, aprovechando sus grandes ventajas (Acero, 2011). Con el propósito de comprobar la solicitación de esfuerzos de los elementos estructurales del conjunto de naves industriales, se realizó una comprobación manual con el paquete computacional, para lo cual se escogieron aquellos elementos que presentaban mayor radio demanda/capacidad, tanto en columnas como en vigas, los mismos que se encuentran tabulados en las Tablas 3 y 4.

Tabla 3. Radio demanda/capacidad en columnas y vigas con modulación entre pórticos H=9 m, L=25 m y B=5 m.

\begin{tabular}{|c|c|c|c|c|c|c|c|}
\hline \multicolumn{4}{|c|}{ Columna } & \multicolumn{4}{|c|}{ Viga } \\
\hline Miembros & Perfil (mm) & $\mathbf{D} / \mathrm{C}_{\mathrm{s}}$ & $\mathbf{D} / \mathrm{C}_{\mathrm{M}}$ & Miembros & Perfil (mm) & $\mathrm{D} / \mathrm{C}_{\mathrm{s}}$ & $\mathrm{D} / \mathrm{C}_{\mathrm{M}}$ \\
\hline PE & $\mathrm{C} 150 \times 5 \times 4$ & 0,849 & 0,838 & PE & $\mathrm{C} 150 \times 5 \times 3$ & 0,647 & 0,639 \\
\hline PI & $\mathrm{C} 150 \times 5 \times 4$ & 0,775 & 0,770 & PI & C $150 \times 5 \times 3$ & 0,702 & 0,693 \\
\hline $\mathrm{CD}$ & $\mathrm{L} 40 \times 4 \times 4$ & 0,906 & 0,893 & $\mathrm{CD}$ & $\mathrm{L} 40 \times 4 \times 5$ & 0,796 & 0,786 \\
\hline $\mathrm{CH}$ & $\mathrm{L} 40 \times 4 \times 4$ & 0,782 & 0,779 & $\mathrm{CH}$ & $\mathrm{L} 40 \times 4 \times 4$ & 0,090 & 0,120 \\
\hline
\end{tabular}

PE: perfil exterior, PI: perfil interior, CD: celosía diagonal, $\mathrm{CH}$ : celosía horizontal, D/C : índice demanda para capacidad obtenido por software, $\mathrm{D} / \mathrm{C}_{\mathrm{M}}$ : índice demanda para capacidad obtenido de forma manual.

Tabla 4. Radio demanda/capacidad en columnas y vigas con modulación entre pórticos H=9 m, L=25 m y B=6 m.

\begin{tabular}{cccccccc}
\hline & \multicolumn{2}{c}{ Columna } & \multicolumn{5}{c}{ Viga } \\
Miembros & Perfil (mm) & D/C & D/C & Miembros & Perfil $(\mathbf{m m})$ & D/C $_{\mathbf{S}}$ & D/C $_{\mathbf{M}}$ \\
\hline PE & $\mathrm{C} 150 \times 5 \times 4$ & 0,914 & 0,908 & $\mathrm{CE}$ & $\mathrm{C} 150 \times 5 \times 3$ & 0,898 & 0,885 \\
$\mathrm{PI}$ & $\mathrm{C} 150 \times 5 \times 4$ & 0,825 & 0,815 & $\mathrm{CI}$ & $\mathrm{C} 150 \times 5 \times 3$ & 0,817 & 0,804 \\
$\mathrm{CD}$ & $\mathrm{L} 40 \times 4 \times 4$ & 0,522 & 0,547 & $\mathrm{CD}$ & $\mathrm{L} 40 \times 4 \times 3$ & 0,866 & 0,859 \\
$\mathrm{CH}$ & $\mathrm{L} 40 \times 4 \times 4$ & 0,862 & 0,856 & $\mathrm{CH}$ & $\mathrm{L} 40 \times 4 \times 3$ & 0,506 & 0,493 \\
\hline
\end{tabular}

PE: perfil exterior, PI: perfil interior, CD: celosía diagonal, $\mathrm{CH}$ : celosía horizontal, D/C : índice demanda para capacidad obtenido por software, $\mathrm{D} / \mathrm{C}_{\mathrm{M}}$ : índice demanda para capacidad obtenido de forma manual.

\section{Interpolación polinómica}

Se utilizó el método de interpolación de alto orden de Lagrange (Pacheco et al., 2012). El análisis numérico que 
define al polinomio de interpolación de Lagrange, correspondiente a $n+1$ valores dados, como aquella función polinomial de grado a lo más $n$ que toma sobre los $n+l$ diferentes valores numéricos $\left\{\mathrm{x}_{0}, \mathrm{x}_{1}, \ldots, \mathrm{x}_{\mathrm{n}}\right\}$, los $\mathrm{n}+\mathrm{l}$ valores dados $\left\{\mathrm{y}_{0}, \mathrm{y}_{1}, \ldots, \mathrm{y}_{\mathrm{n}}\right\}$. Se obtiene un polinomio interpolador como una expresión formal $f(x)=a_{0}+a_{1} x+a_{2} x^{2}+\ldots+a_{0} \cdot x^{n}$ (Sandoval, 2019).

Al suponer conocidos los valores en los $n+1$ puntos $x_{0}, x_{1}, \ldots, x_{n}$ (valores dependientes) y $f_{0}, f_{1}, \ldots, f_{n}$ (valores independientes) de una función; se construyó un polinomio $\mathrm{P}_{\mathrm{n}}(\mathrm{x})$ de grado menor o igual que $\mathrm{n}$, tal como se muestra en la Ecuación 9 según (Sandoval, 2019).

$$
P_{n}(x)=f_{i}, i=0,1, \ldots, n
$$

Al polinomio $\mathrm{P}_{\mathrm{n}}(\mathrm{x})$ así construido, se le denomina polinomio interpolador o polinomio de interpolación. Una posible forma de resolver el problema, fue plantear el siguiente polinomio de grado, indicado en la Ecuación 10:

$$
P_{n}(x)=a_{0}+a_{1} x+a_{1} x^{2}+\cdots+a_{n} x^{n}
$$

Con coeficientes $\mathrm{a}_{\mathrm{i}}, \mathrm{i}=0,1, \ldots, \mathrm{n}$ indeterminados, e imponer que la Ecuación 10 verifique a la Ecuación 9. Esto significa que obtener el polinomio interpolador es equivalente a resolver el sistema de ecuaciones lineales dado en la Ecuación 11:

$$
a_{0}+a_{1} x_{1}+\cdots+a_{n} x_{1}^{n}=f_{1}
$$

\section{Polinomio de Lagrange}

Para esta investigación se utilizó el polinomio de interpolación de Lagrange, ya que este permite generar una función que pasa por todos los puntos, con lo cual se facilita modelar y obtener aproximaciones bastante confiables. Dado el conjunto de puntos $\left(\mathrm{x}_{0} \cdot \mathrm{f}_{0}\right) \cdot\left(\mathrm{x}_{1}, \mathrm{f}_{1}\right) \ldots .\left(\mathrm{x}_{\mathrm{n}} . \mathrm{f}_{\mathrm{n}}\right)$, se considera el polinomio, usando las Ecuaciones 12 hasta 14 (Suárez, 2012$)$.

$$
f_{n}(x)=\sum_{i=0}^{n} f\left(x_{i}\right) L_{i}(x)
$$

Donde $\mathrm{L}_{\mathrm{i}}(\mathrm{x})$ :

$$
L_{i}(x)=\prod_{\substack{j=0 \\ j \neq i}}^{n} \frac{x-x_{j}}{x_{i}-x_{j}}
$$

El polinomio de Lagrange de segundo grado es:

$$
\begin{gathered}
f_{1}(x)=\frac{\left(x-x_{1}\right)\left(x-x_{2}\right)}{\left(x_{0}-x_{1}\right)\left(x_{0}-x_{2}\right)} f\left(x_{0}\right)+ \\
\frac{\left(x-x_{0}\right)\left(x-x_{2}\right)}{\left(x_{1}-x_{0}\right)\left(x_{1}-x_{2}\right)} f\left(x_{1}\right)+ \\
\frac{\left(x-x_{0}\right)\left(x-x_{1}\right)}{\left(x_{2}-x_{0}\right)\left(x_{2}-x_{1}\right)} f\left(x_{2}\right)
\end{gathered}
$$

En este caso $\mathrm{f}_{\mathrm{n}}(\mathrm{x})$ es la "y" interpolada y la "x" es la x para interpolar. Cuantos más datos se tengan en la tabla, se podrá usar un polinomio de mayor grado; en este apartado el polinomio que se obtuvo fue de cuarto grado. Para esta investigación, el valor $\mathrm{f}\left(\mathrm{x}_{\mathrm{i}}\right)$ corresponde a los pesos de cada nave industrial modulados cada 5 y $6 \mathrm{~m}$, mientras que el valor de $\mathrm{L}_{\mathrm{i}}(\mathrm{x})$ es el polinomio de Lagrange, que depende de la luz libre del pórtico, que varía de 15 a $35 \mathrm{~m}$. 


\section{Resultados y Discusión}

El costo de los materiales de una nave industrial para la elaboración de un presupuesto referencial de un proyecto determinado es uno de los factores importantes que influye en la factibilidad y adjudicación del desarrollo del proyecto para la empresa que presupueste una oferta (Guerrero, 2019). La determinación de este parámetro se obtuvo de una forma directa, es decir, el peso de una nave industrial en $\mathrm{kg} / \mathrm{m}^{2}$ indicados en las Figuras 5 y 6, multiplicado por su área. En esta investigación el área osciló entre 225 y $525 \mathrm{~m}_{2}$ para pórticos modulados a cada $5 \mathrm{~m}$, y desde 270 hasta $630 \mathrm{~m}_{2}$ para pórticos modulados a cada $6 \mathrm{~m}$; con estos valores, se obtuvo el peso de material que guarda relación con propiedades de los elementos que se consideró en el diseño.

Con base en los requerimientos y cumpliendo con las normas ASCE (2017), SEI 7-16 (2017), NEC SE AC (2014) y NEC SE DS (2014), en este apartado se presentan los resultados de $\mathrm{kg} / \mathrm{m}^{2}$ alcanzados por las naves industriales como se muestra en la Tabla 5. Así también, se indica el análisis numérico mediante el polinomio de Lagrange, con lo cual se determinaron las ecuaciones que rigen a cada grupo de naves con modulación de 5 y $6 \mathrm{~m}$.

Tabla 5. Pesos totales por metro cuadrado obtenidos para naves con modulación de 5 y 6 m.

\begin{tabular}{|c|c|c|c|c|c|c|c|}
\hline $\begin{array}{c}\text { Luz } \\
\text { libre (m) }\end{array}$ & $\begin{array}{c}\text { Altura } \\
\text { columna (m) }\end{array}$ & $\begin{array}{c}\text { Peso }\left(\mathrm{kg} / \mathrm{m}^{2}\right) \\
\text { modulado a } 5 \text { m }\end{array}$ & $\begin{array}{c}\text { Peso }\left(\mathrm{kg} / \mathrm{m}^{2}\right) \\
\text { modulado a } 6 \text { m }\end{array}$ & $\begin{array}{c}\text { Luz } \\
\text { libre (m) }\end{array}$ & $\begin{array}{c}\text { Altura } \\
\text { columna (m) }\end{array}$ & $\begin{array}{c}\text { Peso }\left(\mathrm{kg} / \mathrm{m}^{2}\right) \\
\text { modulado a } 5 \text { m }\end{array}$ & $\begin{array}{c}\text { Peso }\left(\mathrm{kg} / \mathrm{m}^{2}\right) \\
\text { modulado a } 6 \mathrm{~m}\end{array}$ \\
\hline 15 & 6 & 17,42 & 18,62 & 15 & 8 & 21,01 & 21,63 \\
\hline 20 & 6 & 18,12 & 19,26 & 20 & 8 & 21,64 & 22,11 \\
\hline 25 & 6 & 19,41 & 20,18 & 25 & 8 & 22,48 & 22,96 \\
\hline 30 & 6 & 20,84 & 21,64 & 30 & 8 & 23,79 & 24,37 \\
\hline 35 & 6 & 22,65 & 23,09 & 35 & 8 & 25,49 & 25,92 \\
\hline 15 & 7 & 19,67 & 20,38 & 15 & 9 & 22,60 & 23,24 \\
\hline 20 & 7 & 20,37 & 20,99 & 20 & 9 & 22,94 & 23,74 \\
\hline 25 & 7 & 21,39 & 21,82 & 25 & 9 & 23,70 & 24,37 \\
\hline 30 & 7 & 22,62 & 23,30 & 30 & 9 & 24,84 & 25,49 \\
\hline 35 & 7 & 24,35 & 25,03 & 35 & 9 & 26,21 & 26,96 \\
\hline
\end{tabular}

\section{Resultado de interpolación usando el polinomio de Lagrange}

Una vez conocidos los pesos de la Tabla 5 y aplicando el lenguaje de programación, se conformó las ecuaciones con base en el polinomio de Lagrange para las naves industriales de modulación de 5 y 6 m, con luces que varían cada 5 m y que van desde 15 hasta 35 m respectivamente, como se indica en las Figuras 5 y 6.

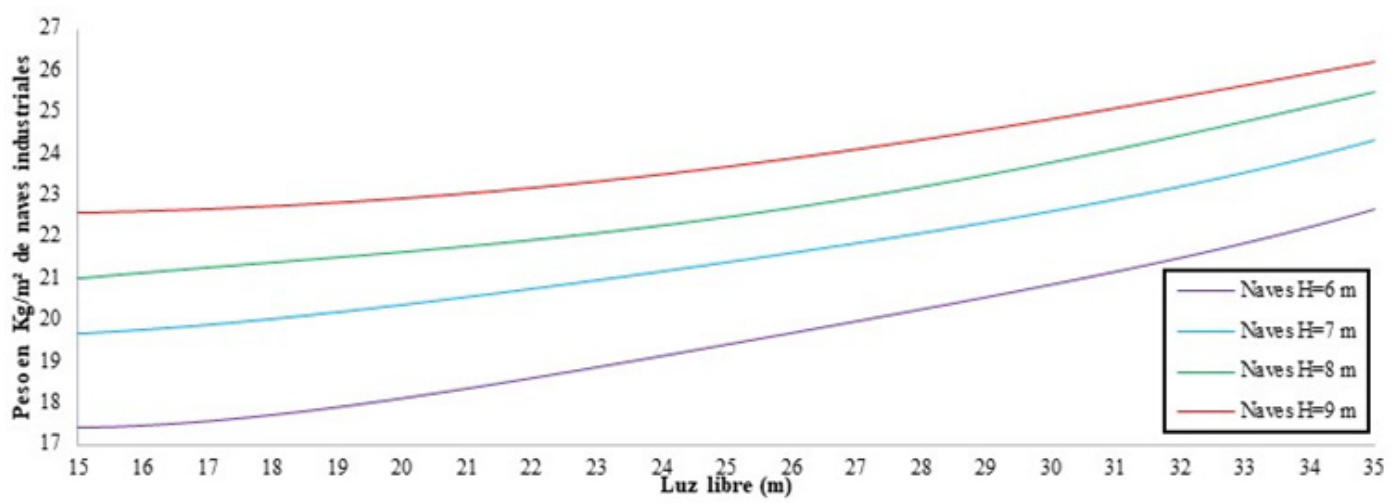

Figura 5. Curvas de interpolación (ábaco) para galpones con modulación de 5 m. 


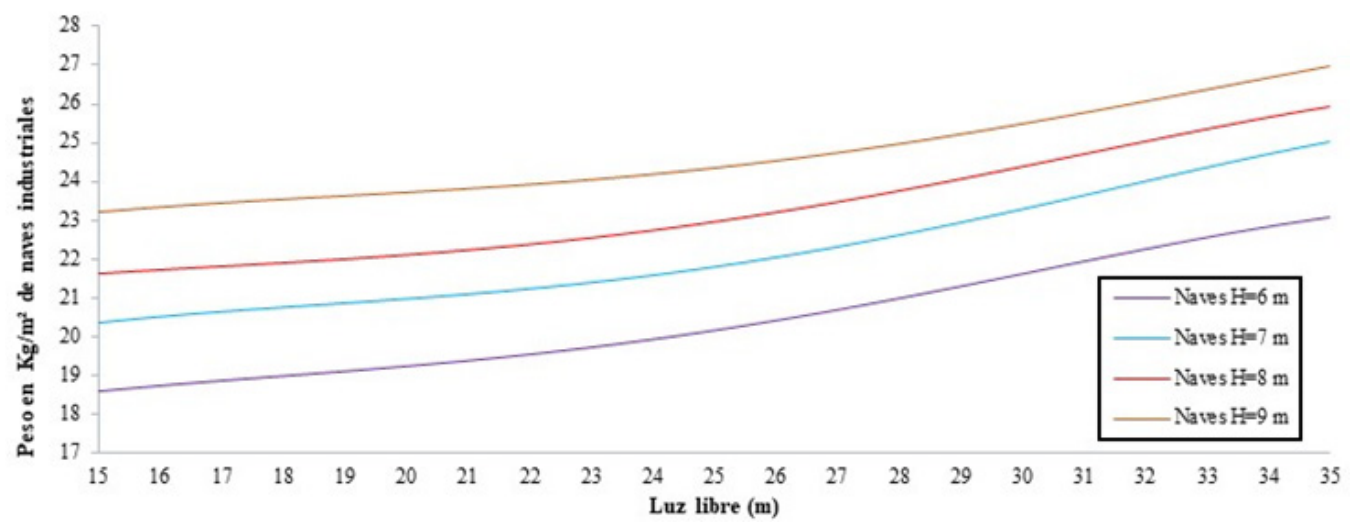

Figura 6. Curvas de interpolación (ábaco) para galpones con modulación de 6 m.

En las Figuras 7 y 8, se presentan los costos de referencias de cada grupo de naves industriales, según modulaciones de 5 y 6 m, respectivamente. El precio de referencia del acero en bobinas laminadas en caliente alcanzó los 499 dólares americanos por tonelada métrica en 2019. Según las estimaciones para el año 2022, esta cifra disminuiría ligeramente hasta situarse en torno a los 463 dólares americanos (Díaz, 2021; Investing, 2021).

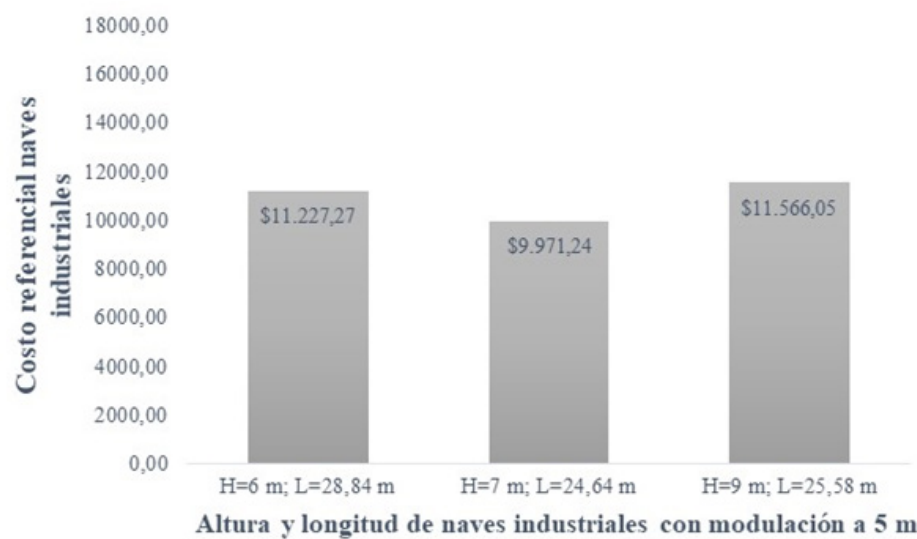

Figura 7. Gráfica de costo referencial de naves industriales moduladas a 5 m (Investing, 2021).

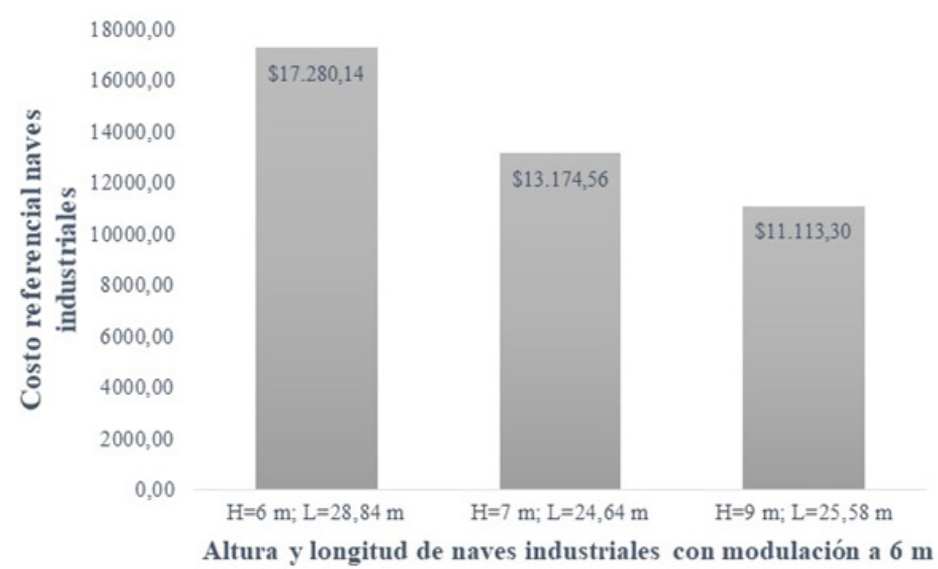

Figura 8. Gráfica de costo referencial de naves industriales moduladas a 6 m (Investing, 2021). 


\section{Determinación del error normalizado porcentual}

Para poder llegar a establecer el valor real a veces es difícil contar con el valor verdadero, para dichos casos, una opción es normalizar el error, empleando la mejor apreciación posible al valor verdadero. Para este estudio el error normalizado porcentual se lo calcula con la expresión dada en la Ecuación 15:

$$
\begin{gathered}
\varepsilon_{t}=\text { errornormalizadoporcentual } \\
E_{t}=\text { valorverdadero-aproximación } \\
\varepsilon_{t}=\frac{E_{t}}{\text { valorverdadero }} \times 100 \%
\end{gathered}
$$

Con el fin de validar los resultados obtenidos en los ábacos de las Figuras 5 y 6; con respecto a la herramienta computacional, en la obtención del peso en $\mathrm{kg} / \mathrm{m}^{2}$ de nave industrial, se asumió que el valor verdadero es aquel que se obtuvo del programa y el valor de aproximación es el valor que se determinó mediante el polinomio de Lagrange. Para comprobación, se evaluaron 6 naves adicionales con valores intermedios no existentes en la Tabla 5, en consecuencia, se

\begin{tabular}{|c|c|c|c|c|c|c|c|}
\hline $\begin{array}{c}\text { B } \\
\text { (m) }\end{array}$ & $\begin{array}{c}\mathbf{H} \\
\text { (m) }\end{array}$ & $\begin{array}{c}\mathbf{L} \\
\text { (m) }\end{array}$ & $\begin{array}{c}\text { Peso con } \\
\text { software }(\mathbf{k g} / \\
\left.\mathbf{m}^{2}\right)\end{array}$ & $\begin{array}{c}\text { Peso con } \\
\text { ecuación }(\mathrm{kg} / \\
\left.\mathbf{m}^{2}\right)\end{array}$ & $\begin{array}{c}\text { Error } \\
(\%)\end{array}$ & $\begin{array}{l}\text { Peso } \\
\text { (kg) }\end{array}$ & $\begin{array}{l}\text { Costo } \\
(\$)\end{array}$ \\
\hline 5 & 6 & 28,84 & 20,49 & 20,50 & 0,05 & 8868,30 & 11227,27 \\
\hline 5 & 7 & 24,64 & 21,31 & 21,31 & 0,00 & 7876,18 & 9971,24 \\
\hline 5 & 9 & 25,58 & 23,80 & 23,81 & 0,04 & 9135,90 & 11566,05 \\
\hline & & & \multicolumn{2}{|c|}{ Media } & 0,03 & & \\
\hline & & & \multicolumn{2}{|c|}{ Desviación } & 0,03 & & \\
\hline
\end{tabular}
obtuvo los resultados presentados en las Tablas 6 y 7.

Tabla 6. Pesos totales por metro cuadrado para valores intermedios obtenidos para galpones con modulación de B=5 m.

\begin{tabular}{|c|c|c|c|c|c|c|c|}
\hline $\begin{array}{r}\text { B } \\
\text { (m) } \\
\end{array}$ & $\begin{array}{c}\mathbf{H} \\
(\mathbf{m}) \\
\end{array}$ & $\begin{array}{l}\mathrm{L} \\
\text { (m) }\end{array}$ & $\begin{array}{c}\text { Peso con } \\
\text { software }(\mathbf{k g} / \\
\left.\mathbf{m}^{2}\right)\end{array}$ & $\begin{array}{c}\text { Peso con } \\
\text { ecuación }(\mathrm{kg} / \\
\left.\mathbf{m}^{2}\right)\end{array}$ & $\begin{array}{c}\text { Error } \\
(\%)\end{array}$ & $\begin{array}{l}\text { Peso } \\
\text { (kg) } \\
\end{array}$ & $\begin{array}{c}\text { Costo } \\
(\$) \\
\end{array}$ \\
\hline 6 & 6 & 33,42 & 22,71 & 22,69 & 0,09 & 13649,40 & 17280,14 \\
\hline 6 & 7 & 26,16 & 22,08 & 22,10 & 0,09 & 10406,45 & 13174,56 \\
\hline 6 & 8 & 21,83 & 22,33 & 22,34 & 0,04 & 8778,28 & 11113,30 \\
\hline & & & \multicolumn{2}{|c|}{ Media } & 0,07 & & \\
\hline & & & \multicolumn{2}{|c|}{ Desviación } & 0,03 & & \\
\hline
\end{tabular}

L: luz libre, H: altura del pórtico, B: distancia entre pórticos.

Tabla 7. Pesos totales por metro cuadrado para valores intermedios obtenidos para galpones con modulación de B=6 m.

L: luz libre, H: altura del pórtico, B: distancia entre pórticos.

Determinación de la proporcionalidad de los parámetros H, L y B Vs. Peso

Con la finalidad de llegar a establecer el nivel de proporcionalidad que existe entre los parámetros antes mencionados (H, L y B) con respecto al peso por cada metro cuadrado del grupo de naves, se obtuvo una gráfica radial, la cual expone que existe una proporcionalidad desde $\mathrm{L}=15 \mathrm{~m}$ hasta $\mathrm{L}=35 \mathrm{~m}$, lo cual se evidencia en la Figura 9. 


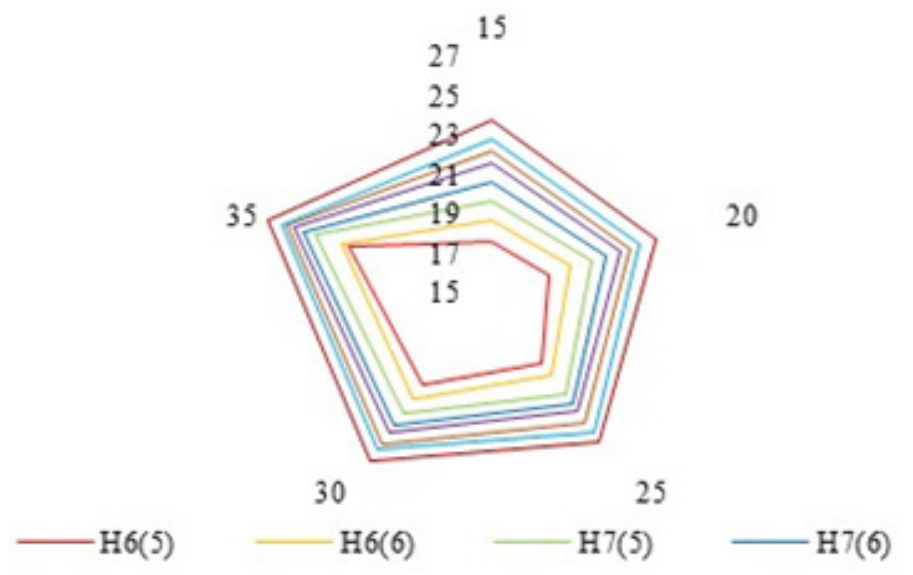

Figura 9. Gráfica radial de la altura libre de columna Vs. el peso por cada metro cuadrado.

\section{Conclusiones}

Al realizar un análisis y diseño estructural utilizando un paquete computacional, de un conjunto de naves industriales con distintos parámetros de longitud, altura y espaciamiento de pórticos, se obtuvieron valores correspondientes al índice entre el peso y área de las estructuras, y al compararlas con los valores obtenidos de polinomio de Lagrange, se encontró un error medio bastante bajo; por lo que se concluye que se puede utilizar simplemente la interpolación indicada en esta investigación con los parámetros antes descritos, para hallar directamente el costo del material acero de las naves industriales.

En lo que respecta al costo de las naves, se deberá tomar en cuenta el valor correspondiente de costo del acero dependiendo únicamente de la zona en la que se vaya a ejecutar el proyecto; en consecuencia, la elaboración de un presupuesto resultará mucho más ágil que el realizar un análisis estructural previo para posteriormente encontrar los costos.

\section{Agradecimiento}

Los autores agradecen a la Universidad Politécnica Salesiana (Quito-Ecuador), en particular a la carrera de ingeniería civil.

\section{Referencias Bibliográficas}

Acero (2020). Dipac Manta [en línea] disponible en: https://dipacmanta.com/perfiles/perfiles-laminados [consulta: 10 marzo 2020].

AISC. (2016). Specification for structural steel buildings. Chicago, Illinois: American Institute of Steel Construction AISC 360-16.

ANSI. (2016). Specification for structural steel buildings. Chicago, Illinois: American Institute of Steel Construction AISC 360-16.

AISI. (2016). North American specification for the design of cold-formed steel structural members. Canada: American Iron and Steel Institute.

Arnal, E., Gutiérrez, F., Montemayor, F. y Achaval, F. (2014). Proyecto y construcción de galpones modulares. 1ํe ed digital. Caracas: PGA Marketing Soluciones C.A.

ASCE. (2017). Minimun design load and associated criteria for buildings and other structures. Virginia: American Society of Civil Engineers (ASCE).

ASTM A1011. (2018). Especificación estándar para acero, láminas y tiras, laminado en caliente, carbono, estructural, 
baja aleación de alta resistencia, baja aleación de alta resistencia con formabilidad mejorada y resistencia ultra alta. West Conshohocken: American Society of Testing Materials (ASTM) International.

A1011M-18ạ. (2018) Especificación estándar para acero, láminas y tiras, laminado en caliente, carbono, estructural, baja aleación de alta resistencia, baja aleación de alta resistencia con formabilidad mejorada y resistencia ultra alta. West Conshohocken: American Society of Testing Materials (ASTM) International.

ASTM A36. (2018). Especificación estándar para acero estructural al carbono. West Conshohocken: American Society of Testing Materials (ASTM) International.

A36M-08. (2018). Especificación estándar para acero estructural al carbono. West Conshohocken: American Society of Testing Materials (ASTM) International.

Blades, S. (2015). Métodos númericos ingeniería civil [en línea]

disponible en: https://cristiancastrop.files.wordpress.com/2010/09/catedra-metodos-numericos-2015-unsch-12.pdf [consulta: 15 marzo 2021].

Cano, P., Imanpour, A. (2020). Evaluation of seismic design methods for steel multi-tiered special concentrically braced frames. Proceedings of the Annual Stability Conference Structural Stability Research Council. Celebrada 10-13 abril 2018 en Baltimore, Maryland.

Crisafulli, F. (2018). Diseño sismo resistente de construcciones de acero. 5 ta ed. Argentina: Asociación Latinoamericana del Acero.

Cuichan, A. (2016). Análisis y diseño de una nave industrial y sus respectivas fosas dentro del taller "Chiriacu" para Ferrocarriles del Ecuador Empresa Pública F.E.E.P. Tesis de grado. Quito: Escuela Politécnica Nacional.

Díaz, A (2021). Statista [en línea] disponible en: https://es.statista.com/estadisticas/634390/precios-del-acero-pormercado-principal/\#: :text=El\%20precio\%20de\%20referencia\%20del,torno\%20a\%20los\%20463\%20d\%C3\%B3lares [consulta: 21 marzo 2021].

EAE (2011). Instrucción de acero estructural EAE. España: Secretaria general técnica. Ministerio de fomento.

Élez, D. (2016). Diseño de estructura básica de nave industrial. Tesis de grado. Valencia: Universitat Politécnica de Valencia.

Garibov, R., Bashirzade, S. (2020). Techno-economic analysis of the same high and different rings Lamella domes using by Lrfd and Asd load combinations. Novateur Publications International Journal of Innovations in Engineering Research and Technology, 7(4), 81-83.

Guerrero, D. (2019). Análisis técnico y económico del diseño por desempeño de edificios con estructura de acero utilizando arriostramientos concéntricos. Gaceta Técnica, 20(1), 41-59. DOI: 10.13140/RG.2.2.20052.86409

Hernández, P. (2015). Determinación de la carga por viento externo en naves con cubierta a dos aguas. Revista Técnica Arquitectura e Ingenieria, 9(1), 1-28.

Investing (2021). Fusion Media Ltd. [en línea] disponible en: https://es.investing.com/commodities/us-steel-coil-futures [consulta: 02 abril 2021].

MathWorks (2019) Matlab (Versión R2020a) [en línea] disponible en: https://matlab.softonic.com [consulta: 08 septiembre 2019].

McCormac, J. (2013). Diseño de estructuras de acero método LRFD. 5ta ed. México: Alfaomega Grupo editor S.A de C.V.

NEC (2014). NEC SE CG. Cargas no sísmicas. Quito: Dirección de Comunicación Social, MIDUVI.

NEC (2014). NEC SE DS. Peligro sísmico. Quito: Dirección de Comunicación Social, MIDUVI.

Pacheco, N., Juárez, C., Martínez, I. (2012). Análisis dinámico de la estabilidad usando interpolación de alto orden. Ingenieria 
Investigación y Tecnología, 13(4), 455.

Pérez, L., Mena, U. (2015). Manual de diseño de obras civiles. Revista Técnica Comisión Federal de Electricidad, 14, $105-106$.

Redroban, D. (2015). Diseño de un galpón industrial aplicando método de elemento finito aplicando el programa ANSYS® para el taller de la empresa metalmecánica. Tesis de grado. Guayaquil: Escuela Superior Politécnica del Litoral.

Sandoval, C. (2019). Métodos numéricos en diferencias finitas para la estimación de recursos de Hardware FPGA en arquitecturas LFSR(n,k) fractales. Ingeniería Investigación y Tecnología, 20(3), 1-4.

Sap 2000. (2016). Integrated solution for structural analysis program. (Versión 19.2.1) [en línea] disponible en: https:// aportesingecivil.com/descargar-sap2000-v20 [consulta: 08 septiembre 2019].

Suárez, M. (2012). Sites.google. [en línea] disponible en: https://sites.google.com/site/metodos0123/unidad-4-ajuste-decurvas-e/4-2-polinomios-de-interpolacion-diferencias-divididas-de-newton-y-de-lagrange [consulta: 15 marzo 2021$].$ 


\section{REVISTA TECNICA}

DE LA FACULTAD DE INGENIERIA

UNIVERSIDAD DEL ZULIA

Vol. 44. №2, Mayo - Agosto, 2021

Esta revista fue editada en formato digital y publicada en Abril de 2021, por el Fondo Editorial Serbiluz, Universidad del Zulia. Maracaibo-Venezuela

www.luz.edu.ve

www.serbi.luz.edu.ve

www.produccioncientificaluz.org 